\title{
Wie erklärt man ein Nash-Gleichgewicht?
}

\author{
Uwe Schäfer \\ Uwe Schäfer studierte von 1989 bis 1994 Diplom-Mathematik an der Universität \\ Karlsruhe und promovierte 1999 ebendort. Seit 1995 arbeitet er als wissenschaftli- \\ cher Mitarbeiter an der Universität Karlsruhe. Hauptsächlich beschäftigt er sich mit \\ Verifikationsnumerik, Optimierung und Geschichte(n) der Mathematik.
}

\section{Einleitung}

Obwohl der Film „A beautiful mind“ von Ron Howard aus dem Jahre 2001 mit dem Satz beginnt, dass Mathematiker den Zweiten Weltkrieg gewonnen hätten, steht nicht die Mathematik im Vordergrund des Films, sondern das Leben des Mathematikers John Forbes Nash jr. Diskutiert man über den Film mit Freunden, Eltern, Lehrerkollegen, Studenten und/oder Schülern, so wird man als Mathematiker oft mit der Frage konfrontiert, was John Forbes Nash jr. denn so Wichtiges geleistet hätte.

Früher oder später steht man dann bei seinen Erläuterungen vor dem Problem, einem Laien das Nash-Gleichgewicht zu erklären. Glücklicherweise ist im Vergleich zu manch einem Ergebnis aus der Reinen Mathematik das Nash-Gleichgewicht relativ elementar. Dennoch sollte man bei seinen Ausführungen nicht mit der mathematischen Definition beginnen. Auch die mathematische Definition lediglich in Worten wiederzugeben, bringt einem Laien das Nash-Gleichgewicht nicht unbedingt näher. Bei Wikipedia, der freien Enzyklopädie im Internet, ist z.B. zu lesen:

Das Nash-Gleichgewicht wird erklärt mit Hilfe einer dritten Person: dem Schlichter. Der Schlichter benutzt die Kenntnis eines Nash-Gleichgewichts in einem ZweiPersonen-Spiel, um die zerstrittenen Parteien zu einer Einigung zu bringen. Ohne die Einführung einer dritten Person wird es schwierig, einem Laien zu erklären, warum sich beide Spieler für ein bestimmtes Nash-Gleichgewicht entscheiden sollten, da ein Zwei-Personen-Spiel mehrere Nash-Gleichgewichte besitzen kann. Der Autor gibt ein einfaches Beispiel eines Zwei-Personen-Spiels, bei dem die so genannten gemischten Strategien ihren Sinn haben, auch ohne mit der Wahrscheinlichkeitstheorie argumentieren zu müssen. 
„Das Nash-Gleichgewicht ist ein zentraler Begriff der mathematischen Spieltheorie. Es beschreibt in Spielen einen Zustand eines strategischen Gleichgewichts, von dem ausgehend kein einzelner Spieler für sich einen Vorteil erzielen kann, indem er allein seine Strategie verändert.“

Bei dieser Definition stellt sich die Frage, wie man in einem Spiel in diesen Zustand gelangt. In [2] ist zu lesen:

„In einem Nash-Gleichgewicht fühlt kein Spieler die Notwendigkeit, sein Verhalten zu ändern, d.h. eine andere als seine (so genannte) Gleichgewichtsstrategie auszuspielen, da er von den anderen Spielern annehmen kann, dass sie mit ihrer Strategie ebenfalls im Gleichgewicht verharren.“

Auch bei dieser Definition stellt sich die Frage, wie man in ein bestimmtes Nash-Gleichgewicht kommt. Man kann nämlich zeigen, dass in einem Spiel mehrere Nash-Gleichgewichte existieren können.

Man umgeht diese Schwierigkeiten, indem man das Nash-Gleichgewicht nicht über seine Definition, sondern über eine Anwendung erklärt. Dazu führen wir eine bestimmte Person ein: den Schlichter.

„Das Nash-Gleichgewicht ist ein Strategiepaar, welches ein Schlichter benutzt,
um in einem Zwei-Personen-Spiel die zerstrittenen Parteien zu einer Einigung
zu bringen.“

Mit dieser Motivation wollen wir nun dem Leser das Nash-Gleichgewicht nahe bringen. Der Leser möge dabei wenigstens mit der Matrix-Vektor-Multiplikation vertraut sein.

\section{Zwei-Personen-Spiele}

Unter einem Zwei-Personen-Spiel verstehen wir ein Spiel, in dem zwei Personen (und zwar Spieler 1 und Spieler 2) gegeneinander spielen. Beide Spieler haben die Möglichkeit, Spielzüge auszuwählen bzw. auf Ereignisse zu setzen. Wir setzen voraus, Spieler 1 könne auf $m$ verschiedene Ereignisse setzen, während Spieler 2 auf $n$ (i. Allg. andere) verschiedene Ereignisse setzen könne. Ein Zwei-Personen-Spiel ist dann gegeben durch zwei $m \times n$ Matrizen

$$
A=\left(\begin{array}{cccc}
a_{11} & a_{12} & \cdots & a_{1 n} \\
a_{21} & a_{22} & \ddots & \vdots \\
\vdots & \ddots & \ddots & a_{m-1 n} \\
a_{m 1} & \cdots & a_{m n-1} & a_{m n}
\end{array}\right), \quad B=\left(\begin{array}{cccc}
b_{11} & b_{12} & \cdots & b_{1 n} \\
b_{21} & b_{22} & \ddots & \vdots \\
\vdots & \ddots & \ddots & b_{m-1 n} \\
b_{m 1} & \cdots & b_{m n-1} & b_{m n}
\end{array}\right) ;
$$

und zwar wie folgt: Setzt Spieler 1 (alles) auf das $i$-te Ereignis und setzt Spieler 2 (alles) auf das $j$-te Ereignis, so ist der Verlust von Spieler 1 beschrieben durch

$$
a_{i j}=e_{i}^{\mathrm{T}} A e_{j},
$$


während der Verlust von Spieler 2 durch

$$
b_{i j}=e_{i}^{\mathrm{T}} B e_{j}
$$

beschrieben wird, wobei $e_{i}$ bzw. $e_{j}$ den $i$-ten bzw. den $j$-ten Standard-Basisvektor bezeichnet. Man nennt die Matrizen $A$ und $B$ auch gerne Verlustmatrizen bzw. Kostenmatrizen.

Oft ist beiden Spielern erlaubt, ihren Einsatz auf die jeweiligen verschiedenen Ereignisse zu verteilen. Zum Beispiel könnte Spieler 1 jeweils die Hälfte seines Einsatzes auf die ersten beiden Ereignisse setzen, und Spieler 2 könnte ein Viertel seines Einsatzes auf das erste Ereignis setzen und den Rest seines Einsatzes auf das $n$-te. Dann würde der Verlust von Spieler 1 beschrieben werden durch

$$
\frac{1}{8} a_{11}+\frac{3}{8} a_{1 n}+\frac{1}{8} a_{21}+\frac{3}{8} a_{2 n}=\left(\begin{array}{llll}
\frac{1}{2} & \frac{1}{2} & 0 & \cdots
\end{array}\right) A\left(\begin{array}{c}
\frac{1}{4} \\
0 \\
\vdots \\
0 \\
\frac{3}{4}
\end{array}\right),
$$

und der Verlust von Spieler 2 würde durch

$$
\frac{1}{8} b_{11}+\frac{3}{8} b_{1 n}+\frac{1}{8} b_{21}+\frac{3}{8} b_{2 n}=\left(\begin{array}{llll}
\frac{1}{2} & \frac{1}{2} & 0 & \cdots 0
\end{array}\right) B\left(\begin{array}{c}
\frac{1}{4} \\
0 \\
\vdots \\
0 \\
\frac{3}{4}
\end{array}\right)
$$

beschrieben werden. Dies führt uns auf den Begriff der gemischten Strategie.

Definition 2.1 Für $m, n \in \mathbb{N}$ setzen wir

$$
\begin{aligned}
S^{m} & :=\left\{x=\left(x_{i}\right) \in \mathbb{R}^{m}: \sum_{i=1}^{m} x_{i}=1, x_{i} \geq 0, i=1, \ldots, m\right\}, \\
S^{n} & :=\left\{y=\left(y_{j}\right) \in \mathbb{R}^{n}: \sum_{j=1}^{n} y_{j}=1, y_{j} \geq 0, j=1, \ldots, n\right\} .
\end{aligned}
$$

Ein $x \in S^{m}$ nennt man eine gemischte Strategie für Spieler 1, und ein $y \in S^{n}$ nennt man eine gemischte Strategie für Spieler 2. Ist speziell $x \in S^{m}$ mit $x_{i}=1$ für ein $i$, so heißt $x$ eine reine Strategie für Spieler 1. Analog ist eine reine Strategie für Spieler 2 definiert. Einer reinen Strategie entspricht also das Setzen des gesamten Einsatzes auf ein Ereignis, während eine gemischte Strategie dem Streuen des Einsatzes entspricht.

Gilt $x \in S^{m}$ und $y \in S^{n}$, so beschreibt $x^{\mathrm{T}} A y$ die Kosten von Spieler 1 und $x^{\mathrm{T}} B y$ die Kosten von Spieler 2. 
Beispiel 2.2 Eine Softwarefirma namens $A$ mit Sitz in Argentinien möge mit einer Softwarefirma namens $B$ mit Sitz in Brasilien zusammenarbeiten. Ein Teil der Zusammenarbeit möge darin bestehen, Daten zu übermitteln. In Argentinien möge es $m$ Netzbetreiber geben, während es in Brasilien deren $n$ gebe. Man habe sich darauf geeinigt, dass die Softwarefirma $A$ die Kosten in Argentinien übernehme, während die Softwarefirma $B$ die Kosten in Brasilien übernehme.

Da die Preise der Netzbetreiber gegeben sind, existieren zwei Kostenmatrizen $A, B \in$ $\mathbb{R}^{m \times n}$ mit folgender Eigenschaft: Nutzt die Softwarefirma $A$ zur Übermittlung der Daten den $i$-ten Netzbetreiber in Argentinien, während die Softwarefirma $B$ zur Übermittlung der Daten den $j$-ten Netzbetreiber in Brasilien wählt, so entstehen in Argentinien für die Softwarefirma $A$ die Kosten $a_{i j}$ und in Brasilien für die Softwarefirma $B$ die Kosten $b_{i j}$. Es stellt sich die Frage, wie die Daten jeweils auf die Netzbetreiber verteilt werden sollen, damit die jeweiligen Kosten gering bleiben.

\section{Das Nash-Gleichgewicht}

Bei einem Zwei-Personen-Spiel stellt sich zwangsläufig die Frage, ob es ein Paar von gemischten Strategien gibt, welches für beide Spieler die Kosten minimiert. Man fragt sich also, ob $\hat{x} \in S^{m}$ und $\hat{y} \in S^{n}$ existieren mit

$$
\hat{x}^{\mathrm{T}} A \hat{y} \leq x^{\mathrm{T}} A y \quad \text { für alle } x \in S^{m}, y \in S^{n}
$$

und

$$
\hat{x}^{\mathrm{T}} B \hat{y} \leq x^{\mathrm{T}} B y \quad \text { für alle } x \in S^{m}, y \in S^{n} .
$$

Das folgende Beispiel zeigt, dass i. Allg. ein solches Paar von gemischten Strategien nicht existiert.

Beispiel 2.3 Es seien

$$
A=\left(\begin{array}{cc}
5 & 0 \\
10 & 1
\end{array}\right) \quad \text { und } \quad B=\left(\begin{array}{cc}
5 & 10 \\
0 & 1
\end{array}\right)
$$

Angenommen, es gebe $\hat{x} \in S^{2}$ und $\hat{y} \in S^{2}$ mit

$$
\hat{x}^{\mathrm{T}} A \hat{y} \leq x^{\mathrm{T}} A y \quad \text { für alle } x \in S^{2}, y \in S^{2}
$$

und

$$
\hat{x}^{\mathrm{T}} B \hat{y} \leq x^{\mathrm{T}} B y \quad \text { für alle } x \in S^{2}, y \in S^{2} .
$$

Wir werden zeigen, dass aus (3) folgt, dass notwendigerweise

$$
\hat{x}=\left(\begin{array}{l}
1 \\
0
\end{array}\right), \quad \hat{y}=\left(\begin{array}{l}
0 \\
1
\end{array}\right)
$$

gelten muss. Zunächst ist offensichtlich, dass für jedes $x \in S^{2}$ und jedes $y \in S^{2}$ wegen $A \geq 0$ die Beziehung $x^{\mathrm{T}} A y \geq 0$ gilt. Da (5) $\hat{x}^{\mathrm{T}} A \hat{y}=0$ erfüllt, ist lediglich noch

$$
\hat{x}^{\mathrm{T}} A \hat{y}=0 \Rightarrow \hat{x}=\left(\begin{array}{l}
1 \\
0
\end{array}\right), \hat{y}=\left(\begin{array}{l}
0 \\
1
\end{array}\right)
$$


zu zeigen. Sei also $\hat{x}^{\mathrm{T}} A \hat{y}=0$. Dann folgen aus

$$
0=\hat{x}^{\mathrm{T}} A \hat{y}=5 \hat{x}_{1} \hat{y}_{1}+10 \hat{x}_{2} \hat{y}_{1}+\hat{x}_{2} \hat{y}_{2}
$$

die drei Bedingungen

$$
5 \hat{x}_{1} \hat{y}_{1}=0, \quad 10 \hat{x}_{2} \hat{y}_{1}=0, \quad \hat{x}_{2} \hat{y}_{2}=0 .
$$

Insbesondere folgt $\hat{x}_{2} \hat{y}_{1}=0$. Wegen $\hat{x}, \hat{y} \in S^{2}$ und (6) erhält man

$$
\hat{y}_{1}=0 \Rightarrow \hat{y}_{2}=1 \Rightarrow \hat{x}_{2}=0
$$

und

$$
\hat{x}_{2}=0 \Rightarrow \hat{x}_{1}=1 \Rightarrow \hat{y}_{1}=0 \text {. }
$$

Somit ist $\hat{y}_{1}=0$ und $\hat{x}_{2}=0$, und es folgt (5). Setzt man (5) in (4) ein, so erhält man einen Widerspruch, denn es ist

$$
\hat{x}^{\mathrm{T}} B \hat{y}=10>0=\left(\begin{array}{ll}
0 & 1
\end{array}\right) B\left(\begin{array}{l}
1 \\
0
\end{array}\right) .
$$

Beispiel 2.3 hat uns gezeigt, dass es bei einem Zwei-Personen-Spiel i. Allg. keine Vorgehensweise gibt, die für beide Spieler die Kosten minimiert. Somit ergibt sich auch kein Vorteil, wenn es den Spielern erlaubt sein sollte, sich gegenseitig abzusprechen.

Man kann ein Zwei-Personen-Spiel, in dem Absprachen erlaubt sind, auch als Verhandlungsspiel deuten, und Beispiel 2.3 zeigt sehr deutlich, warum man sich nicht wundern darf, dass Verhandlungen oft abgebrochen werden, weil sich die Verhandlungspartner nicht einigen können. Einer dritten Person, die die möglicherweise zerstrittenen Parteien zu einer Einigung bringen soll, hilft das Nash-Gleichgewicht.

Definition 2.4 In einem Zwei-Personen-Spiel gegeben durch die Matrizen $A, B \in \mathbb{R}^{m \times n}$ nennt man das gemischte Strategiepaar $(\hat{x}, \hat{y}), \hat{x} \in S^{m}, \hat{y} \in S^{n}$ ein Nash-Gleichgewicht, falls

$$
\begin{array}{ll}
\hat{x}^{\mathrm{T}} A \hat{y} \leq x^{\mathrm{T}} A \hat{y} & \text { für alle } x \in S^{m}, \\
\hat{x}^{\mathrm{T}} B \hat{y} \leq \hat{x}^{\mathrm{T}} B y & \text { für alle } y \in S^{n}
\end{array}
$$

gilt.

Durch die Kenntnis eines Nash-Gleichgewichts $(\hat{x}, \hat{y})$ kann eine dritte Person (nennen wir diese Person Schlichter) in einem Zwei-Personen-Spiel, in dem keine Absprachen erlaubt sind, bzw. in einem Verhandlungsspiel, in dem die beiden Verhandlungspartner miteinander zerstritten sind (und daher nicht miteinander reden), folgendermaßen eine Einigung erreichen:

Der Schlichter geht zu Spieler 1 und sagt:

„Ich weiß, dass Spieler 2 die Strategie $\hat{y}$ wählen wird. Unter dieser Voraussetzung gibt es für Sie nachweislich keine bessere Option als die Strategie $\hat{x}$ zu wählen.“ 
Danach geht der Schlichter zu Spieler 2 und sagt:

„Ich weiß, dass Spieler 1 die Strategie $\hat{x}$ wählen wird. Unter dieser Voraussetzung gibt es für Sie nachweislich keine bessere Option als die Strategie $\hat{y}$ zu wählen.“

Da Spieler 1 und Spieler 2 nicht miteinander reden dürfen bzw. wollen, weiß kein Spieler, ob er als Erster oder als Zweiter vom Schlichter aufgesucht wird, und eine Einigung wird erreicht.

Wir wollen betonen, dass durchaus ein Strategiepaar $(\bar{x}, \bar{y})$ existieren kann mit

$$
\bar{x}^{\mathrm{T}} A \bar{y}<\hat{x}^{\mathrm{T}} A \hat{y} \quad \text { und } \quad \bar{x}^{\mathrm{T}} B \bar{y}<\hat{x}^{\mathrm{T}} B \hat{y} .
$$

Den Hauptnutzen aus der Kenntnis eines Nash-Gleichgewichts $(\hat{x}, \hat{y})$ ziehen also nicht unbedingt die Spieler, sondern den zieht der Schlichter!

Beispiel 2.5 (Das Gefangenen-Dilemma, [5]) Nach einem Raubmord werden zwei verdächtige Personen verhaftet und dem Haftrichter vorgeführt. Der Haftrichter, der die Verdächtigen zum Gestehen bringen will, sieht aber, dass das Beweismaterial sehr dürftig ist, und versucht nun die beiden Verdächtigen gegeneinander auszuspielen:

Falls die Verdächtigen beide gestehen, werden beide mit 5 Jahren Gefängnis bestraft. Gesteht ausschließlich ein Verdächtiger, so wird der Verdächtige, der gestanden hat, frei gelassen und der andere Verdächtige bekommt 10 Jahre Gefängnis (Kronzeugenregelung). Bestreiten die Verdächtigen beide die Tat, so müssen beide 1 Jahr ins Gefängnis auf Grund von Verkehrsdelikten, denn vor der Festnahme war es zu einer wilden Verfolgungsjagd gekommen.

Der Haftrichter lässt die Verdächtigen getrennt in Zellen bringen, so dass sie ihre Entscheidung unabhängig voneinander, d.h. ohne Absprache miteinander, treffen müssen. So sehen sich die Verdächtigen mit einem Zwei-Personen-Spiel konfrontiert, und jeder muss sich für eine Strategie entscheiden.

In diesem Beispiel besteht ein Nash-Gleichgewicht darin, dass die Verdächtigen beide gestehen. Der Haftrichter nützt die Kenntnis dieses Nash-Gleichgewichts geschickt aus: Er sucht nacheinander die beiden Verdächtigen auf und sagt:

„An deiner Stelle würde ich gestehen, denn der andere hat bereits gestanden.“

Dabei weiß kein Verdächtiger, ob er als Erster oder als Zweiter aufgesucht wird. Das Dilemma für die Gefangenen besteht darin, dass der Haftrichter von der Kenntnis des NashGleichgewichts profitiert und nicht die Verdächtigen. Würden die Verdächtigen beide die Tat abstreiten, wäre die Haftstrafe für beide geringer.

Im Unterschied zu Beispiel 2.3 existiert ein Nash-Gleichgewicht immer. Dies hat John Forbes Nash jr. bewiesen ([4]), was die Namensgebung erklärt. Um ein Nash-Gleichgewicht letztlich zu berechnen, löst man ein so genanntes lineares Komplementaritätsproblem; siehe [1]. 
Zum Schluss wollen wir noch anmerken, dass in dem Film „A beautiful mind“ das NashGleichgewicht nicht korrekt erklärt wird; siehe auch [6]. Außerdem gibt es Mathematiker, die der Meinung sind, dass man die mathematischen Leistungen von John Forbes Nash jr. nicht ausschließlich auf das Nash-Gleichgewicht reduzieren sollte; siehe [3].

\section{References}

[1] Lemke, C.E.; Howson, J.T.: Equilibrium points of bimatrix games. J. Soc. Indust. Appl. Math. 12 (1964), $413-423$.

[2] Mehlmann, A.: Wer gewinnt das Spiel? Vieweg Verlag, 1997.

[3] Milnor, J.: John Nash and „A beautiful mind“. Notices Amer. Math. Soc. 45 (1998)10, 1329-1332.

[4] Nash, J.F.: Noncooperative games. Ann. of Math. 54 (1951), 286-295.

[5] Poundstone, W.: Prisoner's Dilemma. University Press, Oxford 1993.

[6] Robinson, S.: The problem with blondes. SIAM News 35 (2002)10, 20.

Uwe Schäfer

Institut für Angewandte Mathematik

Universität Karlsruhe

D-76128 Karlsruhe, Deutschland

e-mail: Uwe.Schaefer@math.uni-karlsruhe.de 